\title{
Editorial for special issue dedicated to VETOMAC XIV—Part 1
}

\author{
Zuzana Dimitrovová ${ }^{1,2}$
}

Published online: 26 November 2019

c) Krishtel eMaging Solutions Private Limited 2019

The 14th International Conference on Vibration Engineering and Technology of Machinery (VETOMAC XIV) was successfully closed in September 2018 at Instituto Superior Técnico of the University of Lisbon under the joint organization of IDMEC-Institute of Mechanical Engineering, Instituto Superior Técnico, University of Lisbon (IDMEC/ IST/UL) and the Department of Civil Engineering, NOVA School of Science and Technology, NOVA University of Lisbon (DEC/FCT/UNL).

The Scientific Program consisted of 6 plenary lectures, around 150 regular presentations and almost 20 poster presentations. Presentations were grouped according to 13 minisymposia and 13 general conference topics. The conference was attended by almost 170 participants from 34 countries. The main objective of the conference was to bring together researchers and engineers devoting their work to vibrationrelated problems in different areas of engineering applications on a common platform.

We strongly believe that VETOMAC XIV had a significant impact on the development of contemporary analytical, numerical and experimental methods in vibration problems, and created an opportunity for opening a forum for discussion and collaboration amongst the participants. Several VETOMAC participants have opted to get their papers published in this journal. From amongst them two special issues are prepared containing the papers that have been accepted in accordance to the review procedure of Journal of Vibration Engineering \& Technologies. I wish to thank the authors for contributing a diverse set of topics covered by their research articles to the special issues. The assistance of the reviewers is greatly appreciated as well. Accepted papers are separated into two groups according to a criterium whether

Zuzana Dimitrovová

zdim@fct.unl.pt

1 Departamento de Engenharia Civil, Faculdade de Ciências e Tecnologia, Universidade NOVA de Lisboa, Lisbon, Portugal

2 IDMEC, Instituto Superior Técnico, Universidade de Lisboa, Lisbon, Portugal they were conceived mainly as theoretical studies or as some practical industrial application. This first issue dedicated to VETOMAC XIV combines together ten selected papers with stronger theoretical background.

The paper by Jiří Náprstek and Cyril Fischer focuses on the theoretical analysis of a tuned mass damper modelled as a heavy ball rolling inside a spherical cavity. The paper is a valuable contribution to the field of nonlinear dynamics and dynamic stability. For such applications, the dominance of the Appell-Gibbs approach over the conventional Lagrangian procedure is emphasized. Several limit trajectories are extracted and physically interpreted; possibilities of further investigation using semianalytical methods are also outlined.

Next two papers are dedicated to energy harvesting. In the one by Max-Uwe Noll et al., the energy harvesting system that consists of a cantilever beam with attached piezoceramic layers is analyzed. The possibility of improving its properties by intentionally introducing nonlinearities is outlined and a new procedure to determine the magnetoelastic forces acting on the beam using the physical laws of the magnetic field is proposed and validated, which is a step towards replacing simple heuristic polynomial magnetic force laws by realistic behavior. In the other paper, written by Yi Li et al., a study on a bistable energy harvester is performed. The paper conducts uncertainty analysis of such harvester to reveal the influence of the uncertain system parameters on the energy harvesting performance. The motivation for the study is the fact, that high-energy oscillations of such harvester cause large amplitude output voltage, substantially influenced by small deviations during manufacturing, which can significantly deviate the output from the designed value.

The paper by Camila A. Xavier da Silva et al. is focused on active modal control of composite beam vibrations with the help of electromagnetic actuators. The linear quadratic regulator solved by linear matrix inequalities was implemented in the control strategy. For the problem definition, the electromagnetic forces behavior was linearized in terms of the displacement and current. The multiobjective optimization problem of system displacements and energy consumption minimization was solved by a new bio-inspired 
optimization strategy, namely by the Multi-objective Optimization Flower Pollination algorithm.

Next paper by Hatim Fakhreddine et al. is dedicated to geometrically non-linear forced vibrations of Euler-Bernoulli multispan beams. The beam is subjected to harmonic forces and can rest on arbitrary number of supports. The geometrical non-linearity is derived from the von Kármán assumptions. The nonlinear transverse displacements of the beam are expanded as a series of the linear vibration modes. The beam total strain and kinetic energies are derived, and by applying Hamilton's principle, the problem is reduced to a nonlinear algebraic system solved by an approximate method.

In the paper by Angelo Tusset et al. the nonlinear dynamics of an Atomic Force Microscopy model in fractional-order is investigated. The level of influence of the derivative order on the dynamics of such a system is identified. To bring the system from a chaotic state to a periodic orbit, the timedelayed feedback control technique for the fractional-order systems was applied. The robustness of the proposed timedelayed feedback control was tested by a sensitivity analysis of parametric uncertainties.

Next paper by Elżbieta Jarzębowska et al. proposes a new method for vibration analysis of dynamic systems with flexible mechanical components, taking into account joint friction and exposed to position and kinematic constraints. A new computationally automated procedure has been developed to generate the system dynamics subjected to programmed constraints. The automated procedure is based on the generalized programmed equations of motion, application of the joint coordinates and homogenous transformation matrices.

In the paper by Sanjin Braut et al. a two-step procedure for rotor-stator partial rub detection is proposed. The possibility of fault detection based on the Instantaneous Angular Speed and the Variational Mode Decomposition is investigated. For this purpose, eight measurements were analyzed on a specially designed test rig. It was concluded that the Variational Mode Decomposition spectrogram of the Instantaneous Angular Speed signal can detect light partial rotor-stator rub condition and draw a clear distinction between no-rubbing and rubbing condition.
The paper by Alexey A. Kireenkov and Sergey I. Zhavoronok is devoted to an analysis of unsteady rolling regimes of pneumatics. An implementation of the theory of multicomponent dry friction is proposed. The main attention is devoted to the construction of analytical models of the combined dry friction accounting for the anisotropy of the dry friction coefficients and the real distribution of normal and tangential contact stresses. The general invariant formulation for the dry friction forces, moments and torque were obtained in case of the simultaneous sliding, spinning, and rolling of the rigid body.

Last paper of this issue presents the work conducted by Kamil Szydło et al. concerned with the comfort of lift passengers. For this purpose, several vibration measurements were taken during travel in a passenger lift. Vibration signals were analyzed by the empirical mode decomposition method and the Hilbert transform. The use of Hilbert spectral analysis enables the isolation of individual signal components and the determination of the dominant frequency, which is then compared with the frequencies important for passengers' comfort.

It is hoped that these special issues dedicated to VETOMAC XIV will give an overview of current research efforts and hence will serve as a useful resource for researchers and students interested in vibration engineering and science. I take this opportunity to thank my colleague, Nuno Maia for accepting to join me as VETOMAC chairperson. I am grateful to Mini-symposia organizers for their contribution to the success of the conference. I would also like to thank for support given by the members of the Steering and Scientific Committee.

I am especially grateful to Jammi Srinivasa Rao, Editor in Chief of "Journal of Vibration Engineering \& Technologies" for giving me the opportunity to organize VETOMAC XIV and conduct the editorial work on these two special issues.

Publisher's Note Springer Nature remains neutral with regard to jurisdictional claims in published maps and institutional affiliations. 